\title{
Typical and atypical development of functional human brain networks: insights from resting-state fMRI
}

\author{
Lucina Q. Uddin ${ }^{1,2 *}$, Kaustubh Supekar ${ }^{3}$ and Vinod Menon ${ }^{1,2,4 *}$ \\ Stanford Cognitive and Systems Neuroscience Laboratory, Department of Psychiatry and Behavioral Sciences, Stanford University School of Medicine, Stanford, \\ CA, USA \\ 2 Program in Neuroscience, Stanford University School of Medicine, Stanford, CA, USA \\ ${ }^{3}$ Graduate Program in Biomedical Informatics, Stanford University School of Medicine, Stanford, CA, USA \\ ${ }^{4}$ Department of Neurology and Neurological Sciences, Stanford University School of Medicine, Stanford, CA, USA
}

\section{Edited by:}

Silvia A. Bunge, University of California Berkeley, USA

\section{Reviewed by:}

Damien Fair, Oregon Health and Science University, USA

Moriah E. Thomason, Stanford

University, USA

\section{*Correspondence:}

Lucina Q. Uddin, Stanford Cognitive and Systems Neuroscience Laboratory,

Department of Psychiatry and

Behavioral Sciences, Stanford

University School of Medicine, 780

Welch Road, Room 201, Stanford, CA 94304, USA.

e-mail: lucina@stanford.edu: Vinod Menon, Stanford Cognitive and Systems Neuroscience Laboratory,

Department of Psychiatry and

Behavioral Sciences, Stanford

University School of Medicine, 780

Welch Road, Room 201, Stanford, CA

94304, USA.

e-mail:menon@stanford.edu
Over the past several decades, structural MRI studies have provided remarkable insights into human brain development by revealing the trajectory of gray and white matter maturation from childhood to adolescence and adulthood. In parallel, functional MRI studies have demonstrated changes in brain activation patterns accompanying cognitive development. Despite these advances, studying the maturation of functional brain networks underlying brain development continues to present unique scientific and methodological challenges. Resting-state fMRI (rsfMRI) has emerged as a novel method for investigating the development of large-scale functional brain networks in infants and young children. We review existing rsfMRI developmental studies and discuss how this method has begun to make significant contributions to our understanding of maturing brain organization. In particular, rsfMRI has been used to complement studies in other modalities investigating the emergence of functional segregation and integration across short and long-range connections spanning the entire brain. We show that rsfMRI studies help to clarify and reveal important principles of functional brain development, including a shift from diffuse to focal activation patterns, and simultaneous pruning of local connectivity and strengthening of long-range connectivity with age. The insights gained from these studies also shed light on potentially disrupted functional networks underlying atypical cognitive development associated with neurodevelopmental disorders. We conclude by identifying critical gaps in the current literature, discussing methodological issues, and suggesting avenues for future research.

Keywords: functional connectivity, brain maturation, resting-state fMRI, cognitive development, autism spectrum disorders, attention-deficit/hyperactivity disorder

\section{INTRODUCTION}

Over the past several decades, refinements in neuroimaging methods have enabled significant insights into human brain development. It is now known that the human brain undergoes a protracted period of development during which changes occur at both the structural and functional levels. Structural neuroimaging studies have shown that while gray matter volume follows a regionally specific inverted U-shaped trajectory, white matter volume shows protracted increases with development (Lenroot and Giedd, 2006). This general principle affects sensorimotor and higher-order association cortices at different time points in development (Gogtay et al., 2004). These regressive (gray matter loss) and progressive (white matter increases) changes are related to cognitive development (Casey et al., 2005). Age-related changes in cortical thickness have also been reported to show regional specificity (Sowell et al., 2004). Exactly how these structural changes impact functional brain maturation is less well understood.

Abbreviations: fMRI, functional magnetic resonance imaging; rsfMRI, restingstate fMRI; ICA, independent component analysis; ReHO, regional homogeneity; ADHD, attention-deficit/hyperactivity disorder; ASD, autism spectrum disorder; TS, Tourette syndrome; DMN, default mode network; DTI, diffusion tensor imaging.
In general, functional brain maturation is much more difficult to study than structural maturation because differences in observed brain activation can arise from differences in cognitive effort, experience, and strategy (Casey et al., 2005). This presents a significant challenge for understanding the development of functional brain networks. The existing literature suggests that brain activation during a particular cognitive task tends to progress from more diffuse to more focal with age. Durston et al. (2006) were the first to describe this notion of diffuse-to-focal changes in activation patterns in a longitudinal study of cognitive control. They demonstrated that over time, participants revealed an increase of magnitude of activation in key frontal areas with concomitant attenuation of activation in more widespread, nonspecific brain regions. They interpreted this to reflect development of more efficient processing. There is also evidence to suggest that with development, there are decreased demands on prefrontal cortex, with increased reliance on posterior brain regions (Rivera et al., 2005). However, there is often a complex relationship between changes in brain activation related to performance differences versus those related to brain maturation, and it is often difficult to match children on performance (Tamm et al., 2002). Even in cases where children, adolescents and adults are matched on accuracy, 
it is often impossible to control for differences in reaction time (Kwon et al., 2002). Thus, while researchers typically attempt to control for performance-related issues in their studies, this is not always readily accomplished. The complex relationships between learning, experience, and development therefore make it difficult to uncover principles governing functional brain development, and complicate the interpretation of reported differences in brain activation between children and adults.

Resting-state fMRI (rsfMRI) offers a novel framework for studying the development of functional brain circuits, and in particular for better understanding the large-scale organization of the developing brain. This method is now increasingly used to complement traditional task-based fMRI. rsfMRI involves collecting functional imaging data from participants as they lay in the MRI scanner, typically fixating gaze on a cross-hair or with eyes closed, and refraining from engaging in any specific cognitive task. Since initial reports of coherent spontaneous low-frequency fluctuations in BOLD signal within the somatomotor system in the absence of a specific task (Biswal et al., 1995), it has been widely demonstrated that brain networks that are engaged during cognitive tasks can also be reliably identified during resting states (Smith et al., 2009, see Fox and Raichle, 2007 for review). Resting-state functional connectivity uses spontaneous synchronized fluctuations in BOLD signal to determine tightly coupled functional brain networks independent of task-induced correlations. These spontaneous fluctuations are posited to act to organize, coordinate, and maintain functional brain systems (Fox and Raichle, 2007; Raichle, 2010), and bias information processing (Greicius and Menon, 2004). The advantages of using rsfMRI in pediatric and clinical populations are that functional brain organization can be examined independent of task performance, and a full dataset can be collected in as little as 5 min (Van Dijk et al., 2009). Functional connectivity measures derived from rsfMRI data are particularly useful in studying age-related changes in the wiring of neural networks (Stevens, 2009; Supekar et al., 2009). Withinand between-subject measures computed from rsfMRI are quite consistent and reproducible (Damoiseaux et al., 2006; Shehzad et al., 2009). Because the resting-state scanning procedure places a minimal cognitive burden on the participant, and requires relatively little time in the scanner compared to task fMRI studies, data can be collected from low-functioning and very young populations. A recent study of the fMRI success rate of children and adolescents reports that the success rate for completing an entire battery of experimental fMRI runs varied between 50 and $59 \%$ in patient populations such as attention-deficit/hyperactivity disorder (ADHD) and autism spectrum disorder (ASD), and 69\% for typically developing children (Yerys et al., 2009). As every pediatric neuroimaging researcher knows, excessive motion and floor task performance are issues that hinder successful scanning of young children, and account for significant data loss. The use of rsfMRI data obviates the need for long scan sessions as well as concerns regarding task performance, thus is particularly well suited for studies of typical and atypical brain development. In addition, certain widely adopted methodological approaches to analyzing rsfMRI data [e.g., independent component analysis (ICA), discussed below] are quite successful at removing motion artifacts, resulting in significantly less data loss.
The first study to use rsfMRI to study brain development was conducted in 2000. Curiously, subsequent rsfMRI studies in children did not appear until 2006. The use of rsfMRI in developmental studies is clearly still in its infancy, and our review of the emerging literature is necessarily constrained by this limitation. Table 1 summarizes current studies that have used rsfMRI to examine the typical and atypical development of functional brain networks. We begin with summaries of major findings regarding brain maturation from rsfMRI studies in neurotypical infants, children and adolescents. Within each section of the review, studies are grouped by analysis method employed, as the specific methods can have important implications for the interpretation of findings. We next review contributions of rsfMRI to the study of neurodevelopmental disorders, focusing primarily on ADHD and ASD. Next, methodological issues related to the acquisition and analyses of rsfMRI data for developmental studies are discussed. We conclude by highlighting outstanding issues in the field and suggesting avenues for future research.

\section{TYPICAL DEVELOPMENT: STUDIES IN INFANTS AND YOUNG CHILDREN \\ ROI SEED-BASED ANALYSIS}

Region-of-interest (ROI) seed-based analysis is one of the two most widely used methods in analysis of rsfMRI data. The method is a hypothesis-driven approach that typically involves choosing one or more ROIs and examining their whole-brain functional connectivity, often using a regression or correlation model. Kiviniemi et al. (2000) used this method in the first reported study of developing functional networks using rsfMRI. The authors studied children between 3 months and 10 years of age. During the scan participants were sedated with thiopental. Using one representative voxel time course from primary visual cortex, the authors demonstrated correlated activity within striate and extrastriate cortex as well as occipital cortices adjacent to primary visual cortex. This study demonstrated that children as young as 3 months of age have stable visual networks, and that they can be detected in rsfMRI data.

Ethical concerns preclude the use of sedation in studies of typical development. The first study to take advantage of natural sleep as a method to study brain function in young children was conducted by Redcay et al. (2007). The authors used an ROI-based approach and reported functional connectivity between superior temporal gyrus and medial and lateral prefrontal regions, as well as between occipital and parietal regions, in 2- to 4-year-old typically developing children during low-level auditory and visual stimulation. The extent to which these results reflect task-related versus intrinsic functional connectivity is at present unclear. Nonetheless, this study highlights the utility of imaging during natural sleep to obtain high quality $\mathrm{fMRI}$ data from very young children, which would otherwise be impossible to collect in this age range.

Lin et al. (2008) took advantage of rsfMRI during natural sleep to study functional connectivity of primary motor, sensory, and visual areas in children between 2 weeks and 2 years of age while they were asleep. This study found that the percentage of brain volume exhibiting resting functional connectivity, and the strength of resting functional connectivity, increased non-uniformly from 2 weeks to 2 years of age. The percentage of brain volume that showed resting functional connectivity in sensorimotor cortices was larger than that 
Table 1 | Summary of resting-state fMRI studies in infants, children and adolescents, including neurodevelopmental disorders.

\begin{tabular}{|c|c|c|c|c|}
\hline Ages studied & Authors & Population & Analyses & Brain regions examined \\
\hline $4.29 \pm 2.56$ years & Kiviniemi et al. (2000) & Typically developing & ROI-based FC & Visual cortex \\
\hline $\begin{array}{l}\text { Visual experiment: } 46.4 \pm 6.7 \\
\text { months; Auditory experiment: } \\
46.9 \pm 9.7 \text { months }\end{array}$ & Redcay et al. (2007) & Typically developing & ROI-based FC & Superior temporal gyrus, occipital cortex \\
\hline $\begin{array}{l}\text { Range: } 39 \text { weeks and } 1 \text { day to } \\
44 \text { weeks and } 2 \text { days (gestational } \\
\text { age at MRI: } 41 \text { weeks) }\end{array}$ & Fransson et al. (2007) & Typically developing & ICA & $\begin{array}{l}\text { Somatosensory and motor cortices, } \\
\text { temporal/inferior parietal cortex, posterior } \\
\text { lateral and midline parietal cortex/lateral } \\
\text { aspects of the cerebellum, medial and } \\
\text { lateral sections of anterior prefrontal cortex }\end{array}$ \\
\hline 7-9 years; $10-15$ years; $19-31$ years & Fair et al. (2008) & Typically developing & $\begin{array}{l}\text { ROI-based FC, } \\
\text { graph theory }\end{array}$ & Default mode network \\
\hline $\begin{array}{l}\text { Children: } 10.6 \pm 1.5 \text { years; } \\
\text { Adolescents: } 15.4 \pm 1.2 \text { years; } \\
\text { Adults: } 22.4 \pm 1.2 \text { years }\end{array}$ & Kelly et al. (2009) & Typically developing & ROI-based FC & Cingulate-based networks \\
\hline $12-30$ years & Stevens et al. (2009) & Typically developing & $\begin{array}{l}\text { ICA, causality } \\
\text { estimates }\end{array}$ & Whole-brain \\
\hline $\begin{array}{l}\text { Working memory: } 7-11 \text { years; Rest: } \\
\text { 9-12 years }\end{array}$ & Thomason et al. (2008) & Typically developing & ICA & Default mode network \\
\hline $\begin{array}{l}\text { Children: } 7-9 \text { years, mean } 8.6 \text {; } \\
\text { Adolescents: } 10-15 \text { years, mean } \\
\text { 11.9; Adults: } 20-31 \text { years, mean } \\
24.1\end{array}$ & Fair et al. (2007) & Typically developing & Graph theory & $\begin{array}{l}\text { Thirty-nine putative task-control regions } \\
\text { within fronto-parietal and cingulo-opercular } \\
\text { networks }\end{array}$ \\
\hline 7-31 years & Fair et al. (2009) & Typically developing & Graph theory & $\begin{array}{l}\text { Cingulo-opercular, fronto-parietal, cerebellar, } \\
\text { and default mode networks }\end{array}$ \\
\hline $\begin{array}{l}\text { Children: 7-9 years; Adult: 19-22 } \\
\text { years }\end{array}$ & Supekar et al. (2010) & Typically developing & $\begin{array}{l}\text { ICA, white } \\
\text { matter fiber } \\
\text { tracking }\end{array}$ & Default mode network \\
\hline $\begin{array}{l}\text { ADHD: } 13.91 \pm 0.35 \text { years; Control: } \\
13.20 \pm 0.56 \text { years }\end{array}$ & Tian et al. (2006) & ADHD & ROI-based FC & Dorsal anterior cingulate cortex \\
\hline $\begin{array}{l}\text { ADHD: } 13.37 \pm 1.49 \text { years; Control: } \\
13.32 \pm 0.95 \text { years }\end{array}$ & Cao et al. (2006) & ADHD & $\mathrm{ReHo}$ & Whole-brain \\
\hline $\begin{array}{l}\text { ADHD: } 13.34 \pm 1.44 \text { years; Control: } \\
\text { age-matched within } 0.5 \text { year }\end{array}$ & Zhu et al. (2008) & ADHD & $\begin{array}{l}\text { ReHo, Fisher } \\
\text { discriminative } \\
\text { analysis }\end{array}$ & Whole-brain \\
\hline $\begin{array}{l}\text { ADHD: } 13.0 \pm 1.4 \text { years; Control: } \\
13.1 \pm 0.6 \text { years }\end{array}$ & Zang et al. (2007) & ADHD & ALFF & Whole-brain \\
\hline $\begin{array}{l}\text { ADHD: } 13.48 \pm 1.11 \text { years; Control: } \\
13.19 \pm 0.49 \text { years }\end{array}$ & Tian et al. (2008) & ADHD & RSAI & Whole-brain \\
\hline $\begin{array}{l}\text { ADHD: } 13.3 \pm 1.4 \text { years; Control: } \\
13.2 \pm 1.0 \text { years }\end{array}$ & Cao et al. (2009) & ADHD & ROI-based FC & Putamen \\
\hline $\begin{array}{l}\text { ADHD: } 34.9 \pm 9.9 \text { years; Control: } \\
31.2 \pm 9.0 \text { years }\end{array}$ & $\begin{array}{l}\text { Castellanos et al. } \\
\text { (2008) }\end{array}$ & ADHD & ROI-based FC & Cingulate-based networks \\
\hline
\end{tabular}


Table 1 | (Continued)

\begin{tabular}{|c|c|c|c|c|}
\hline Ages studied & Authors & Population & Analyses & Brain regions examined \\
\hline $\begin{array}{l}\text { ADHD: } 34.9 \pm 9.9 \text { years; Control: } \\
31.2 \pm 9.0 \text { years }\end{array}$ & Uddin et al. (2008) & ADHD & NetHo & Default mode network \\
\hline $\begin{array}{l}\text { ASD: } 24 \pm 10.6 \text { years; control: } \\
24 \pm 9.0 \text { years }\end{array}$ & $\begin{array}{l}\text { Cherkassky et al. } \\
\text { (2006) }\end{array}$ & ASD & ROI-based FC & $\begin{array}{l}\text { Posterior cingulate cortex, ventral anterior } \\
\text { cingulate cortex, precuneus, paracentral } \\
\text { lobule, bilateral medial/middle prefrontal } \\
\text { cortex, bilateral inferior parietal cortex, } \\
\text { bilateral parahippocampal gyrus, bilateral } \\
\text { inferolateral temporal cortex (insula) }\end{array}$ \\
\hline $\begin{array}{l}\text { ASD: } 26.5 \pm 12.8 \text { years; Control: } \\
27.5 \pm 10.9 \text { years }\end{array}$ & Kennedy et al. (2008) & ASD & ROl-based FC & $\begin{array}{l}\text { Task-positive (dorsal attention) network, } \\
\text { task-negative (default mode) network }\end{array}$ \\
\hline $\begin{array}{l}\text { ASD: } 26 \pm 5.93 \text { years; TD: } 27 \pm 6.1 \\
\text { years }\end{array}$ & Monk et al. (2009) & ASD & ROl-based FC & Default mode network \\
\hline $\begin{array}{l}\text { ASD: } 15 \pm 1.45 \text { years; TD: } 16 \pm 1.44 \\
\text { years }\end{array}$ & Weng et al. (2010) & ASD & ROl-based FC & Default mode network \\
\hline 8 years & Paakki et al. (2010) & ASD & $\mathrm{ReHo}$ & Whole-brain \\
\hline $\begin{array}{l}\text { TS: } 12.70 \text { years }(9.92-15.83) ; \text { TD: } \\
12.69 \text { years }(10.42-15.75)\end{array}$ & Church et al. (2009) & Tourette syndrome & ROI-based FC & $\begin{array}{l}\text { Thirty-nine putative task control regions } \\
\text { within fronto-parietal and cingulo-opercular } \\
\text { networks }\end{array}$ \\
\hline $\begin{array}{l}\text { MDD: } 16.5 \pm 0.95 \text { years; TD: } \\
16.8 \pm 1.5 \text { years }\end{array}$ & Cullen et al. (2009) & Depression & ROI-based FC & $\begin{array}{l}\text { Subgenual ACC, amygdala, supragenual } \\
\text { ACC }\end{array}$ \\
\hline $12.2 \pm 2.1$ years & Thomason et al. (2009) & $\begin{array}{l}\text { BDNF met-allele } \\
\text { carriers }\end{array}$ & ROI-based FC & $\begin{array}{l}\text { Default mode network, executive control } \\
\text { network, salience network }\end{array}$ \\
\hline
\end{tabular}

$A D H D$, attention-deficit/hyperactivity disorder; ALFF, amplitude of low-frequency fluctuation; ASD, autism spectrum disorder; FC, functional connectivity; ICA independent component analysis; MDD, major depressive disorder; NetHo, network homogeneity; ReHo, regional homogeneity; ROI, region-of-interest; RSAI, resting-state activity index; TD, typically developing.

in the visual cortex for subjects 2 weeks of age and for 1-year-olds, but not for the 2-year-olds, suggesting that functional connectivity in sensorimotor networks precedes that in the visual networks. This study highlights the utility of rsfMRI for examining development of specific functional systems in infants and very young children. The findings shed light on different developmental trajectories of distinct cortical networks underlying sensorimotor and visual processing.

\section{ICA-BASED ANALYSIS}

ICA, unlike ROI-based analysis, is a model-free, data-driven approach whereby four-dimensional fMRI data is decomposed into a set of independent one-dimensional time series and associated three-dimensional spatial maps which describe the temporal and spatial characteristics of the underlying signals or components (Beckmann et al., 2005). ICA is currently a widely used method for analyzing rsfMRI data (Calhoun et al., 2008).

In 2007, Fransson et al. studied resting-state activity in the brains of sedated sleeping infants under 1 year of age. Using ICA, five distinct networks were identified in the infant brain. These consistent networks comprised (1) primary visual areas, (2) bilateral somatosensory and motor cortices, (3) bilateral temporal/inferior parietal cortex including primary auditory cortex, (4) posterior lateral and midline parts of the parietal cortex and lateral aspects of the cerebellum, and (5) medial and lateral sections of anterior prefrontal cortex. Intriguingly, these networks represent a subset of those previously demonstrated to exist in the adult brain (Damoiseaux et al., 2006; De Luca et al., 2006), with the notable exception that the infant brain appeared to lack a component along the anterior-posterior direction, despite the fact that transcallosal functional connectivity was intact at this very young age. The authors specifically noted that they did not detect an equivalent of the default mode network (DMN) in infants (Fransson et al., 2007). The DMN is so named due to its uniquely high metabolic resting activity (Raichle et al., 2001) and characteristic deactivation during challenging cognitive tasks (Shulman et al., 1997). The network includes posterior cingulate cortex (PCC), medial prefrontal cortex (MPFC), and bilateral inferior parietal lobule (IPL, Raichle et al., 2001). While the functions of this network are widely debated, there is growing evidence that it is involved in high level self-related (e.g., autobiographical and prospective memory) (Buckner and Carroll, 2007) or social cognitive (e.g., theory of mind and moral cognition) processes (Harrison et al., 2008). Fransson et al. (2007) suggest that the absence of this network in infants may be related to both immature anterior-posterior white matter connectivity and immature development of these cognitive processes.

In another study, Liu et al. (2008) studied functional connectivity of somatomotor areas in 1-year-old infants. They used ICA to detect a sensorimotor network, and reported greater intrahemispheric connectivity than interhemispheric connectivity within sensorimotor areas. This finding is in line with the Fransson et al. (2007) 
study. This suggests a universal principle guiding development of large-scale brain systems, namely that the development of intrahemispheric connectivity within local functional circuits precedes the development of interhemispheric connectivity.

\section{GRAPH THEORETICAL AND NETWORK ANALYSIS}

Graphs are data structures which have nodes and edges between the nodes (Bondy and Murty, 1976). Graph theoretical metrics such as clustering coefficient, path length, degree, and centrality provide quantitative measures to characterize large-scale networks represented as a graph (see Bullmore and Sporns, 2009 for a detailed review of various graph metrics and their interpretation). In a graphical representation of a brain network, a node corresponds to a brain region while an edge corresponds to the functional interactions between two brain regions. In recent years, there has been increasing interest in the use and application of graph metrics to characterize large-scale brain networks. This is in part due to the emergence and availability of rsfMRI data. In addition to the advantages described above, resting-state data allow, for the first time, simultaneous in vivo examination of all brain regions and the intrinsic interactions among them. More importantly, patterns of resting-state correlations are thought to reflect functional architecture of the brain (Hagmann et al., 2008; Margulies et al., 2009; van den Heuvel et al., 2009). Several studies have used graph theoretical approaches to characterize large-scale brain networks using rsfMRI. In adults, converging evidence from studies suggests that the adult brain has a robust and efficient small-world architecture comprised of hubs with a high degree connectivity and a modular structure (see Bullmore and Sporns, 2009 for a comprehensive review). Developmental studies are beginning to examine how these network metrics change with age and cognitive skill.

In a developmental context, Gao et al. (2009) used a combination of graph theoretical analyses and ICA to examine a large-scale brain network (DMN) in healthy 2-week to 2-year-old sleeping children. They found a primitive and incomplete DMN in 2-week-olds, followed by an increase in the number of brain regions exhibiting connectivity at 1 year of age. The $\mathrm{DMN}$ at 2 years of age began to resemble that observed in adults, in that it included MPFC, PCC/ retrosplenial (PCC/Rsp), IPL, lateral temporal cortex, and hippocampus. As previously discussed, this network is thought to subserve self-related and social cognitive processes (Uddin et al., 2007; Spreng et al., 2009). The authors then used a measure called "betweenness" centrality, a graph theoretical measure of node importance, to show that the PCC/Rsp nodes showed the most elevated centrality measure for all age groups. The PCC/Rsp node was consistently observed in both age groups, suggesting that this region may form a "hub" even at the earliest developmental stage. They also found that the MPFC showed elevated centrality measures, though not as high as the PCC/Rsp. They suggest that the MPFC emerges as a potential secondary hub starting at the age of 1 .

\section{TYPICAL DEVELOPMENT: STUDIES IN OLDER CHILDREN, ADOLESCENTS AND YOUNG-ADULTS ROI SEED-BASED ANALYSIS}

Fair et al. (2008) used ROI-based analyses to examine differential connectivity of the ventromedial prefrontal cortex (vmPFC) in a group of 7- to 9-year-old children compared to 21- to 31-year-old adults. Children showed marked decreases in connectivity along the anterior-posterior dimension, between the vmPFC and PCC, compared to adults. To further examine differences in brain network structure between children and adults, the time series associated with 13 nodes within the DMN (such as left and right lateral parietal cortex, left and right parahippocampal gyrus, MPFC, and retrosplenial cortex) were correlated with each other. Comparison of correlation matrices generated from child and adult rsfMRI data revealed that nodes within the DMN were sparsely connected in children, and strongly functionally connected in adults. Interestingly, in this age range, interhemispheric functional connections between homotopic regions were reported to be strong in children, as in adults.

In addition to providing detailed information about the connectivity profiles of individual nodes, ROI-based analyses have aided in delineating regionally specific developmental changes within functionally heterogeneous brain regions. In a recent study, Kelly et al. (2009) examined the development of five functionally distinct cingulate-based networks in children (age 11 years), adolescents (age 15) and young-adults (age 22). These networks have been previously characterized in adults (Margulies et al., 2007), and underscore the heterogeneous connectivity of subregions within the cingulate cortex. This study found that children demonstrated a more diffuse pattern of correlation with voxels proximal to the seed ROI, whereas adults exhibited more focal patterns of functional connectivity, as well as a greater number of correlated voxels at long distances from the seed ROI. Adolescents exhibited intermediate patterns of connectivity between the children and adults. Interestingly, connectivity of networks associated with social and emotional functions [based in subgenual anterior cingulate cortex (ACC) and vmPFC] exhibited the greatest developmental effects, while connectivity of networks associated with motor control and conflict monitoring (dorsal ACC) did not differ greatly between the three groups (Kelly et al., 2009). This study demonstrates that rsfMRI data recapitulates two organizational principles of development that have previously been proposed, namely the shift from diffuse to focal activity with age (Durston et al., 2006), and the development of motor systems preceding the development of systems underlying higher cognition (Chugani et al., 1987).

\section{ICA-BASED ANALYSIS}

Using an ICA analysis in conjunction with "causal density" estimates, Stevens et al. (2009) characterized functional networks in 100 participants ranging in age from 12 to 30 years. The authors identified 13 components of interest and used Granger causality estimates to examine interactions between these networks. They report that mutual influences among networks decreased with age, and speculate that this reflects stronger within-network connectivity and more efficient between-network influences with development. Interestingly, they also found age-related reductions in the strength of interaction between lateral prefrontal-parietal (executive control) circuits and networks identified as resembling the DMN. They suggest that more segregated functioning of these sets of networks may allow greater processing flexibility.

Thomason et al. (2008) identified the DMN in 7- to 12-year-old children using both rsfMRI and examination of task-induced deactivations. They report overlap between brain regions comprising the DMN as identified by ICA applied to rsfMRI data, and regions 
comprising the DMN as identified by load-dependent deactivation during a working memory task. These DMN regions identified by two methods overlapped with the regions previously reported in adults. They also found that cognitive measures collected outside the scanner correlated with BOLD decreases during the working memory tasks.

\section{GRAPH THEORETICAL AND NETWORK ANALYSIS}

In order to understand functional brain development, it is critical to investigate and characterize the underlying developmental processes that produce systematic changes in functional brain organization. Fair et al. (2008) were the first to examine this developmental process using rsfMRI. In a related study, they focused on a larger network comprised of 39 cortical regions involved in task-control (Fair et al., 2007a). This study reported a developmental process trend towards "segregation" (general decrease in connectivity strength) between regions close in anatomical space and "integration" (increased connectivity strength) between specific regions distant in Euclidean space, within the 39-node network. More generally, the authors of these two studies argue that the organization of large-scale functional brain networks shifts from a local anatomical emphasis in children to a more distributed architecture in young-adults (Fair et al., 2009).

In contrast to examining developmental process within circumscribed network nodes, Supekar et al. (2009) investigated age-related functional connectivity changes across 90 cortical and subcortical regions that spanned the entire brain. More specifically, they analyzed inter-regional functional connectivity changes within this 90-node whole-brain network in relation to distance between the regions. The inter-regional distance was measured using quantitative diffusion tensor imaging-based white matter tractography, rather than Euclidean distance between regions as used in the studies by Fair et al. (2007a). Additional analyses further revealed that adults have weaker short-range functional connectivity and stronger longrange functional connectivity than do children. Taken together, the studies by Fair et al. (2007a, 2009), Kelly et al. (2009), and Supekar et al. (2009) suggest a developmental process of greater functional segregation in children and greater functional integration in adults at the whole-brain level, as well as in specific networks such as the attentional control network and the DMN.

Understanding how the functional organization of the human brain matures and evolves from childhood to adolescence to adulthood is fundamentally important for gaining insights into the maturation of brain function. As described earlier, the graph theoretical approach is well suited for characterizing functional organization of the brain at multiple levels of granularity. At the global "wholebrain" level, Supekar et al. (2009) reported that both 7- to 9-year-old children and 19- to 22-year-old adults exhibit small-world architecture characterized by high clustering and short path lengths. A small-world architecture was also revealed in a study of multiple functional networks involving distributed nodes conducted by Fair et al. (2009). These studies indicate that at the global level, the human brain is comprised of sub-networks of densely connected nodes, mostly connected by short path lengths. More importantly, these studies indicate that this robust organization is conserved from early childhood to adulthood. At the sub-network level, however, Supekar et al. (2009) found significant differences in inter-regional connectivity patterns between children and adults. Notably, in children, subcortical areas were more strongly connected with primary sensory, association, and paralimbic areas, whereas adults showed greater cortico-cortical connectivity between paralimbic, limbic, and association areas (Figure 1). This finding is in line with previous work demonstrating greater reliance on subcortical structures in children during cognitive tasks (Luna et al., 2001; Thomas et al., 2004). Taken together these studies indicate that while the global functional organization of the human brain is similar in 7- to 9year-old children and adults, at the sub-network level, brain connectivity undergoes significant reorganization with development.

In summary, studies examining functional brain organization in infants, children, and adolescents have revealed consistent findings with respect to the development of long distance connectivity and regional functional specialization. The ability to study very young children at critical developmental milestones is an advantage of the rsfMRI approach, which allows for the in vivo examination of intrinsic functional architecture across the entire brain. However, most of these studies have been conducted in older children, adolescents and adults, and thus to date there is little known regarding how global or local network organization changes during the important developmental period from infancy to young childhood.

\section{NEURODEVELOPMENTAL DISORDERS: STUDIES IN CHILDREN AND ADULTS \\ OVERVIEW}

In addition to enabling unique insights into typical brain development, rsfMRI has been used to explore potentially altered functional connectivity associated with neurodevelopmental disorders. Unfortunately, the majority of these studies have not focused on infants and young children, an issue that is particularly pressing in early impact disorders such as ASD. Nonetheless, the theoretical and methodological progress that has resulted from studies of older children and adults is paving the way for similar studies in younger populations.

\section{ATTENTION-DEFICIT/HYPERACTIVITY DISORDER}

Several recent studies have focused on ADHD, although few consistent findings have emerged. An early study in adolescents found that patients with ADHD showed more significant resting-state functional connectivity between dorsal ACC and thalamus, cerebellum, insula, and brainstem (Tian et al., 2006). Using an approach termed "regional homogeneity" ( $\mathrm{ReHo}$ ), which measures the similarity of a voxel's time series to its neighbors, it was reported that children with ADHD showed decreased ReHo in frontal-striatal-cerebellar circuits and increased ReHo in the occipital cortex (Cao et al., 2006), suggesting disordered functional organization in circuits previously implicated in structural brain imaging studies of ADHD (Giedd et al., 2001). Classifiers based on ReHo measures have been used to discriminate children with ADHD from controls with $85 \%$ accuracy (Zhu et al., 2008). Zang et al. (2007) showed that children with ADHD had decreased amplitude of low-frequency fluctuations (ALFF: $0.01-0.08 \mathrm{~Hz}$ ) in the right inferior frontal cortex, left sensorimotor cortex, and bilateral cerebellum, and increased ALFF in the right ACC, left sensorimotor cortex, and bilateral brainstem. Using a measure incorporating ReHo and variance of LFF, Tian et al. (2008) found that adolescents with ADHD showed greater 


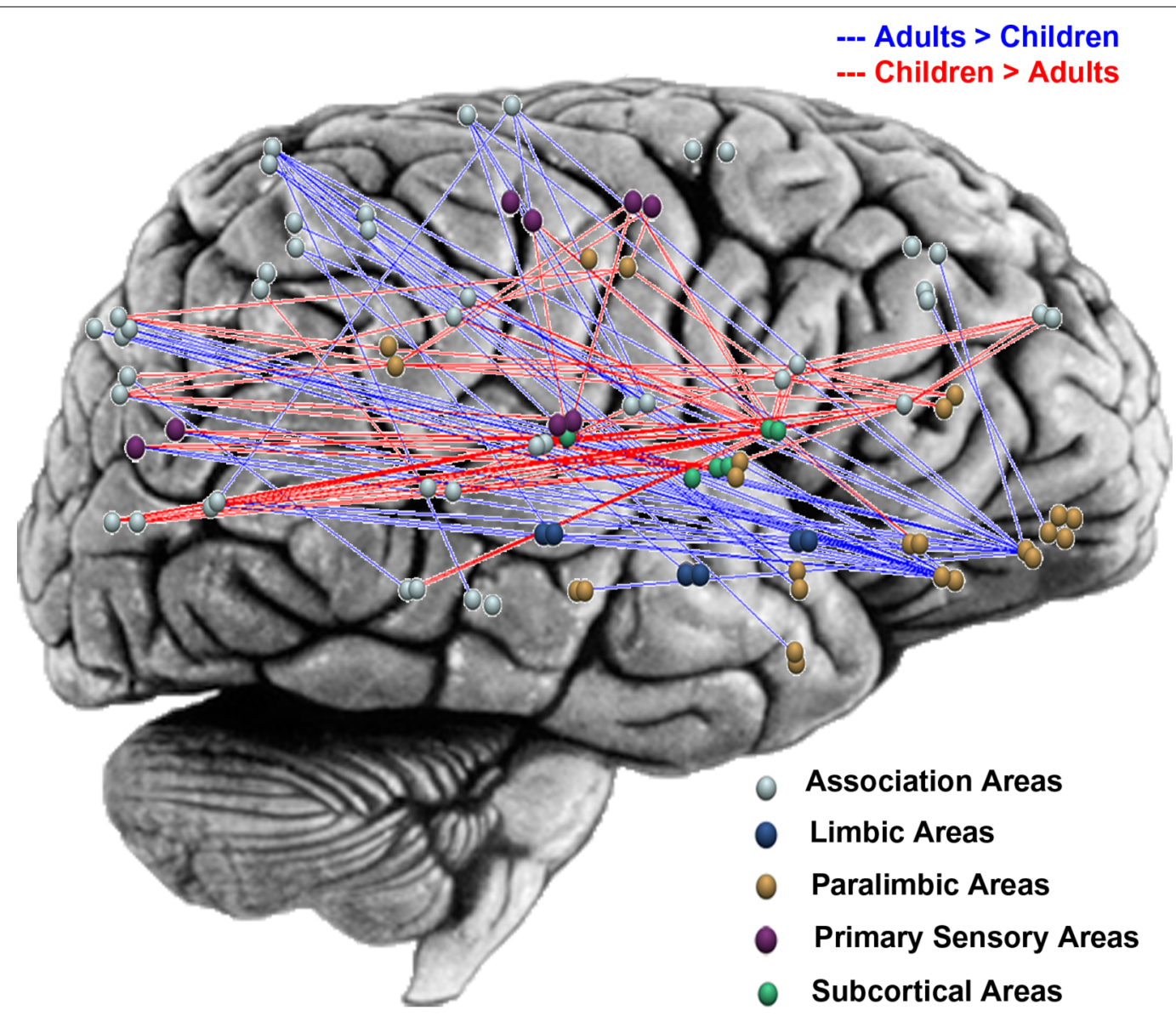

FIGURE 1 | Graphical representation of developmental changes in functional connectivity along the posterior-anterior and ventral-dorsal axes, highlighting higher subcortical connectivity (subcortical nodes are shown in green) and lower paralimbic connectivity (paralimbic nodes are shown in gold) in children, compared to young-adults. Brain regions are plotted using the $y$ and $z$ coordinates of their centroids (in millimeter) in the $\mathrm{MNI}$ space. Four hundred and thirty pairs of anatomical regions showed significantly higher correlations in children and 321 pairs showed significantly higher correlations in young-adults ( $p<0.005$, FDR corrected). For illustration purposes, the plot shows differential connectivity that was most significant, 105 pairs higher in children (indicated in red) and 53 higher in young-adults (indicated in blue) ( $p<0.0001$, FDR corrected). Adapted from Supekar et al. (2009). resting-state activity than controls in basic sensory areas (bilateral BA 17/18/19, left BA 3, left BA 22, and bilateral thalamus). Yet another study reported putamen-specific functional connectivity abnormalities in ADHD, with group differences in putamen and cortical-striatal-thalamic circuits (Cao et al., 2009). Though intriguing, very few replicable findings have emerged from these studies, perhaps due to the relatively small sample sizes and heterogeneity of symptomatology in the patients examined.

$\mathrm{ADHD}$ is known to be associated with attentional lapses (Castellanos et al., 2005), thus recent studies have begun to focus on understanding how circuitry within the ACC may contribute to the symptoms of the disorder. A study of 20 adults with ADHD and 20 matched controls found decreases in negative correlations between the anterior cingulate and precuneus/ PCC regions as well as decreases in connectivity between precuneus and other default mode network components, including vmPFC (Castellanos et al., 2008). This finding was bolstered by similar results utilizing a different measure on the same dataset, which found reduced "network homogeneity" within the DMN in ADHD, particularly between the precuneus and other DMN regions (Uddin et al., 2008). These findings are in line with a theory positing that spontaneous patterns of very low frequency coherence within the DMN may intrude into periods of active task-specific processing, producing periodic fluctuations in attention that compete with goal-directed activity in ADHD (Sonuga-Barke and Castellanos, 2007).

\section{AUTISM SPECTRUM DISORDERS}

Autism is another major neurodevelopmental disorder that has long been associated with disruptions in brain connectivity (Frith, 2004). Surprisingly, to date there have been no published reports of rsfMRI studies in children with ASD. However, a few studies have used this method to study adolescents and adults with ASD. Cherkassky et al. (2006) used an ROI-based approach to demonstrate functional underconnectivity in anterior-posterior connections in ASD. ASD is associated with altered socioemotional responses, which have been linked to the DMN. Kennedy and Courchesne (2008) showed, in a mixed group of adolescents and adults, disrupted intrinsic connectivity in the DMN, but not the executive control network. Another 
recent study replicated this finding of reduced DMN connectivity, and further demonstrated that restricted and repetitive behaviors in ASD were correlated with the degree of connectivity between the PCC and right parahippocampal gyrus (Monk et al., 2009). This group went on to examine DMN connectivity in adolescents with ASD. They also found that relative to controls, adolescents with ASD showed weaker connectivity in nine of the eleven areas of the DMN that were analyzed. Additionally, poorer social skills and increases in restricted and repetitive behaviors and interests correlated with weaker connectivity, whereas poorer verbal and non-verbal communication correlated with stronger connectivity in multiple areas of the DMN. Compared to their study of adults with ASD, these findings indicate that adolescents with ASD show even weaker connectivity in the DMN (Weng et al., 2010). Paakki et al. (2010) used the ReHo approach to study adolescents with ASD, and found that compared with the controls, the subjects with ASD had significantly decreased ReHo in right superior temporal sulcus region, right inferior and middle frontal gyri, right insula and right postcentral gyrus. Significantly increased ReHo was shown in left inferior frontal and anterior subcallosal gyrus (Paakki et al., 2010). A study in neurotypical adults found that functional connectivity between the anterior insula and ACC was related to social responsiveness (Di Martino et al., 2009). Future research is needed to examine how reduced functional connectivity between specific brain regions impacts symptom severity in young children and adolescents with ASD, and how these reductions influence deficits in performance on tasks involving social information processing.

While rsfMRI studies relevant to understanding the neural basis of ASD are still in their infancy, they highlight the utility and value of this approach. In addition, these studies have identified previously understudied candidate brain regions and large-scale networks of interest. In particular, we believe that the study of relationships between networks involved in self-related and social cognition (DMN), externally oriented attention (executive control) and switching between them should be particularly prioritized in future studies of ASD (Uddin and Menon, 2009).

\section{OTHER NEURODEVELOPMENTAL DISORDERS AND GENETIC EFFECTS}

Tourette syndrome (TS) is another pediatric disorder that has recently been studied using rsfMRI methods. Church et al. (2009) found that a fronto-parietal network involved in rapid, adaptive online control was weaker in adolescents with TS. Adolescents with major depressive disorder have decreased functional connectivity in a subgenual ACC-based neural network that includes the supragenual ACC, right medial frontal cortex, the left inferior and superior frontal cortex, superior temporal gyrus, and the insular cortex, areas involved in mediating emotion processing (Cullen et al., 2009).

Intriguingly, specific genetic polymorphisms have also been shown to affect resting-state functional connectivity measures in children (Thomason et al., 2009). BDNF gene variants, associated with alterations in brain anatomy and memory, appear to affect functional connectivity. Thomason et al. (2009) found a reduction in hippocampal and parahippocampal to cortical connectivity in met-allele carriers within each of three resting networks (the default mode, executive, and paralimbic networks), as well as increased connectivity to amygdala, insula and striatal regions in met-carriers, within the paralimbic network. Thus, genetic differences can contribute to functional connectivity differences at the systems-level. How these differences in functional connectivity influence memory function remains to be investigated.

\section{METHODOLOGICAL ISSUES AND CHALLENGES}

One of the primary challenges in pediatric neuroimaging is the fact that the procedure requires participants to remain motionless for an extended period of time. As this is particularly difficult for young children, especially for clinical populations such as children with ADHD or ASD, fMRI data often contains significant motion artifacts, resulting in up to $50 \%$ data loss in some cases (Yerys et al., 2009). Two main solutions to this problem are currently implemented, one at the data collection level and one at the data processing level. Data acquisition protocols often include a "mock scanner" training session, during which children practice lying still in a scanner-like mockup. Post data acquisition, artifact correction algorithms can also be implemented to remove motion-related spikes in the data (Mazaika et al., 2007, 2009). Currently, these procedures are not universally utilized, and considerable variability exists between research centers with respect to criteria for inclusion of data containing motion artifacts. As previously discussed, one advantage of rsfMRI is that it requires very little time in the scanner, and thus can be more easily collected from young participants. In addition, resting-state data is often analyzed using ICA, as summarized in the studies reviewed above. This analysis method may be helpful in effectively isolating motion-related artifacts as distinct independent components (Beckmann et al., 2005).

A second issue concerns the use of varied instructions to the participant during acquisition of rsfMRI data. By definition, resting-state signal reflects activity during a task-free ("rest") state. Accordingly, most of the developmental studies reviewed here have used data collected while the participants were not performing any task. Although these data indeed reflect resting conditions, a closer look indicates that participants were either instructed to fixate on a cross-hair, or keep their eyes open while viewing a blank screen, or keep their eyes closed for the duration of the scan. A recent study, however, indicated that resting-state connectivity within the DMN and attention network was significantly diminished in participants with eyes closed, compared to eyes open or a fixation condition (Van Dijk et al., 2009).

An important related issue is the use of "rest blocks" within task data to conduct analyses typically performed on pure resting-state data. Fair et al. (2007b) used rest blocks extracted from interleaved experimental task data in studies investigating developmental changes in intrinsic functional connectivity. Cherkassky et al. (2006) used a similar approach to investigate resting-state functional connectivity in autism, as reviewed. Another group used residual signal obtained by regressing out task-evoked effects from an event-related task to study functional connectivity within fronto-parietal resting-state networks (He et al., 2007). Given the inherent resting-state connectivity differences even within various resting states, it is more than of academic interest to investigate how well these datasets relate to pure rsfMRI data. Fair et al. (2007b) investigated this issue by comparing resting-state functional connectivity measures from data obtained from (1) residual data from event-related designs, (2) continuous resting-state data, (3) resting 
data that was interleaved between task blocks, and (4) simulated interleaved resting data that was created using the continuous resting data set. They reported that the residual data set, the interleaved dataset, and the simulated interleaved dataset were mostly similar to the continuous resting state dataset, with some differences. The greatest caveats on interpretation of functional connectivity results were placed on the use of event-related data residuals (Fair et al., 2007b).

Another issue concerns the current lack of studies examining test-retest reliability of resting-state data collected for developmental studies. While the patterns of resting-state functional connectivity have been shown to be reproducible across adult participants and scans (van de Ven et al., 2004; Damoiseaux et al., 2006), there is limited evidence about their test-retest reliability, particularly for the pediatric populations. In adults, Shehzad et al. (2009) reported that the test-retest reliability of resting-state functional connectivity was high for significant positive correlations and relatively low for non-significant and negative correlations. Furthermore, they showed that the reliability for DMN connectivity was higher compared to task positive networks. Meindl et al. (2009) reported similar reproducibility results. They observed, across three scan sessions, higher reliability for DMN correlations and lower for non-DMN correlations. These results were further confirmed by Zuo et al. (2010a,b). Although the results of these studies appear promising, Honey et al. (2009) recently raised concerns about the test-retest reliability of rsfMRI data. For individual participants, they observed overall low reproducibility across scans for restingstate correlations between 998 ROIs. All of these previous studies have been conducted in adults, therefore it is not at all known to what extent this issue affects studies of development.

Yet another issue pertains to statistical power or lack thereof in pediatric resting-state studies. Most studies to date have had included relatively small numbers of participants. Although analyses of power and sample sizes have been reported for conventional task-based functional neuroimaging studies (Desmond and Glover, 2002), similar power analyses have not yet been applied to rsfMRI. This is particularly important for studies of typical and atypical pediatric populations, which are inherently highly heterogeneous. Ideally, along with statistical power analyses, a clinical pediatric resting-state study should be comprised of a relatively homogenous group, as well as a homogenous well-matched control group for meaningful interpretations and better comparability of findings across studies.

Finally, it is now well documented that raw rsfMRI data is contaminated by motion artifacts, scanner artifacts, and physiological noise (Biswal et al., 1995; Lowe et al., 1998; Cordes et al., 2000, 2001). To remove this noise, researchers have used a gamut of techniques including, but not limited to, spatial smoothing (to improve signalto-noise ratio), temporal filtering (to remove signal contributed by physiological sources such as cardiac and respiratory cycles) (De Luca et al., 2006; Supekar et al., 2009), whole-brain signal regression (to account for noise sources such as motion, cardiac, and respiratory signals that globally influence the signal) (Desjardins et al., 2001), and regressing out cardiac and respiratory signals acquired in the scanner (to minimize unwanted physiological variations) (Birn et al., 2008; Chang and Glover, 2009; Chang et al., 2009). Although these techniques are fairly effective in removing noise, they raise some concerns regarding the interpretation of the preprocessed data. For example, regressing out whole-brain signal has shown to introduce negative correlations (Murphy et al., 2009; Smith et al., 2009; Van Dijk et al., 2009), though there is no reason to believe that all reported negative correlations are artifactual (Fox et al., 2009). Preprocessing including global signal correction has been shown to increase connection specificity. However, as negative correlations can be induced by this procedure, there is reason to interpret the directionality of these relations with caution especially when global regression is used (Weissenbacher et al., 2009). While these issues are a concern for all rsfMRI studies, not just those exploring developmental issues, the field has not yet reached a consensus as to how to best minimize the effects of noise on these analyses.

\section{SUMMARY AND FUTURE DIRECTIONS}

In this review, we have summarized the current status of research utilizing rsfMRI to examine the typical and atypical development of functional brain circuits. Several key principles of human brain development are beginning to emerge from this literature. In particular, these studies suggest that intrahemispheric connectivity develops before interhemispheric connectivity (Fransson et al., 2007; Liu et al., 2008), and that anterior-posterior connectivity is slowest to develop (Kelly et al., 2009). Sensorimotor networks emerge early in infancy and appear to develop well before visual networks (Lin et al., 2008). Whether this applies to other sensory systems remains to be investigated. Such investigations may provide important insights and have implications for cognitive development, specifically with respect to an infant's early exploration of the world.

Beyond infancy, there is converging evidence from multiple studies suggesting that by age 7-9 children manifest a similar "small world" type of functional architecture at the whole-brain level as adults (Fair et al., 2009; Supekar et al., 2009). However, the organization of individual functional sub-networks as well as their interactions have a protracted developmental time course. This process is characterized by a number of developmental features. First, children have stronger and more abundant connections between subcortical and cortical regions, while in young-adults, connections among cortical regions were more prominent. Second, the brains of young-adults are more hierarchically organized, with more regions involved in larger and longer-distance clusters of activity. Third, the development of large-scale brain networks is characterized by weakening of short-range functional connectivity and strengthening of long-range functional connectivity. Taken together, these findings suggest that the dynamic process of overconnectivity followed by pruning, which rewires connectivity at the neuronal level, also operates at the systems level and helps reconfigure and rebalance cortical and subcortical connectivity in the developing brain (Supekar et al., 2009).

Several methodological issues remain to be addressed before the field can move forward. These are thoroughly discussed and reviewed in another contribution to this Special Issue (Cole et al., 2010). It is our belief that the two main methodological approaches discussed here (ICA and seed-ROI based correlation) each make important contributions to the study of intrinsic brain architecture, and can be used in a complementary fashion to understand global and local functional properties of the developing brain. 
At present, it is unknown how and to what extent changes in functional connectivity are related to structural brain maturation. Directions for future research include integrating rsfMRI with diffusion tensor imaging (DTI) to investigate how the maturity of specific fiber tracts relates to the maturation of cognitive function and skill acquisition. A recent study examined developmental changes in DMN connectivity using a multimodal imaging approach by combining rsfMRI, voxel-based morphometry and diffusion tensor imaging-based tractography. The authors found that the DMN undergoes significant developmental changes in functional and structural connectivity, but these changes are not uniform across all DMN nodes. Critically, this study found that functional connectivity in children can reach adult-like levels despite weak structural connectivity (Supekar et al., 2010). Improved multimodal analysis of anatomy and connectivity will allow us to better characterize the heterogeneous development and maturation of functional brain networks.

It has recently been demonstrated that one possible function of resting-state functional connectivity is to support the consolidation of previous experience. Lewis et al. (2009) showed that visual perceptual learning, an example of adult neural plasticity, modified the resting covariance structure of spontaneous activity between networks engaged by the task, and that the observed changes correlated with the degree of perceptual learning. The complex relationships between cognitive performance and integrity of resting-state networks is only beginning to be explored, and future work in this area will have particular significance for developmental psychologists and neuroscientists.

Additional future directions include incorporating knowledge of genetics into rsfMRI studies, as well as continued investigations into relationships between functional connectivity and cognition and behavior. The field would particularly benefit from longitudinal studies that would allow tracking of development of connectivity

\section{REFERENCES}

Beckmann, C. F., DeLuca, M., Devlin, J.T., and Smith, S.M. (2005). Investigations into resting-state connectivity using independent component analysis. Philos. Trans. R. Soc. Lond., B, Biol. Sci. 360, 1001-1013.

Birn, R. M., Smith, M. A., Jones, T. B., and Bandettini, P. A. (2008). The respiration response function: the temporal dynamics of fMRI signal fluctuations related to changes in respiration. Neuroimage 40, 644-654.

Biswal, B., Yetkin, F. Z., Haughton, V. M., and Hyde, J. S. (1995). Functional connectivity in the motor cortex of resting human brain using echoplanar MRI. Magn. Reson. Med. 34, 537-541.

Biswal, B. B., Mennes, M., Zuo, X. N., Gohel, S., Kelly, C., Smith, S. M., Beckmann, C. F., Adelstein, J. S., Buckner, R. L., Colcombe, S., Dogonowski, A. M., Ernst, M., Fair, D., Hampson, M., Hoptman, M. J., Hyde, J. S., Kiviniemi, V. J., Kötter, R., Li, S. J., Lin, C. P., Lowe, M. J.,
Mackay, C., Madden, D. J., Madsen, K. H., Margulies, D. S., Mayberg, H. S., McMahon, K., Monk, C. S., Mostofsky, S. H., Nagel, B. J., Pekar, J. J., Peltier, S. J., Petersen, S. E., Riedl, V., Rombouts, S. A., Rypmam B., Schlaggar, B. L., Schmidt, S., Seidler, R. D., Siegle, G. J., Sorg, C., Teng, G. J., Veijola, J., Villringer, A., Walter, M., Wang, L., Weng, X. C., Whitfield-Gabrieli, S., Williamson, P., Windischberger, C., Zang, Y. F., Zhang, H. Y., Castellanos, F. X., and Milham, M. P. (2010). Toward discovery science of human brain function. Proc. Natl. Acad. Sci. U.S.A. 107, 4734-4739.

Bondy, J. A., and Murty, U. S. R. (1976). Graph Theory with Applications. New York: American Elsevier Pub. Co.

Buckner, R. L., and Carroll, D. C. (2007). Self-projection and the brain. Trends Cogn. Sci. 11, 49-57.

Bullmore, E., and Sporns, O. (2009). Complex brain networks: graph theoretical analysis of structural and functional systems. Nat. Rev. Neurosci. 10, 186-198.

within individuals. Mapping the developmental trajectory of functional brain organization will be greatly facilitated by a longitudinal approach. Lastly, elucidating brain organization related to neurodevelopmental disorders is perhaps the arena in which rsfMRI can make the greatest contributions. Very young and low-functioning children who might not otherwise be able to tolerate the scanner environment may be able to participate in a resting-state scan with a 5-min duration. Such data can be used to derive brain-based biomarkers that may in the future lead to early diagnosis and thus the development of more efficient and targeted treatments and therapies.

The use of rsfMRI for studying typical and atypical brain development is still in its infancy. Critically, its potential for synthesis and uncovering general organizational principles underlying functional brain development remain largely untapped. Current efforts to pool resources and data across multiple sites will in the future result in larger sample sizes, which are particularly critical for addressing clinical developmental questions. These data-sharing efforts have already produced interesting insights into brain organization in typically developing adults (Biswal et al., 2010). With rapid methodological improvements in rsfMRI, and the use of larger, more refined samples, we can expect to see rapid progress in the use of rsfMRI for addressing important research questions in developmental systems neuroscience.

\section{ACKNOWLEDGMENTS}

This work was supported by a Mosbacher Postdoctoral Fellowship and a National Institute of Mental Health Career Development Award (K01MH092288) to Lucina Q. Uddin, as well as grants from the National Institutes of Health (NS058899, HD047520, HD059205, HD057610) to Vinod Menon, and the National Science Foundation (BCS/DRL 0449927) to Vinod Menon. The content is solely the responsibility of the authors and does not necessarily represent the official views of the NIMH or the NIH.

Calhoun, V.D., Kiehl, K. A., and Pearlson, G. D. (2008). Modulation of temporally coherent brain networks estimated using ICA at rest and during cognitive tasks. Hum. Brain Mapp. 29, 828-838.

Cao, Q., Zang, Y., Sun, L., Sui, M., Long X., Zou, Q., and Wang, Y. (2006). Abnormal neural activity in children with attention deficit hyperactivity disorder: a resting-state functional magnetic resonance imaging study. Neuroreport 17, 1033-1036.

Cao, X., Cao, Q., Long, X., Sun, L., Sui, M., Zhu, C., Zuo, X., Zang, Y., and Wang, Y. (2009). Abnormal resting-state functional connectivity patterns of the putamen in medication-naïve children with attention deficit hyperactivity disorder. Brain Res. 1303, 195-206.

Casey, B. J., Galvan, A., and Hare, T. A. (2005). Changes in cerebral functional organization during cognitive development. Curr. Opin. Neurobiol. 15, 239-244.

Castellanos, F. X., Margulies, D. S., Kelly, C., Uddin, L. Q., Ghaffari, M., Kirsch,
A., Shaw, D., Shehzad, Z., Di Martino, A., Biswal, B., Sonuga-Barke, E. J. S., Rotrosen, J., Adler, L. A., and Milham, M. P. (2008). Cingulate-precuneus interactions: a new locus of dysfunction in adult attention-deficit/hyperactivity disorder. Biol. Psychiatry 63, 332-337.

Castellanos, F. X., Sonuga-Barke, E. J., Scheres, A., Di Martino, A., Hyde, C., and Walters, J. R. (2005). Varieties of attention-deficit/hyperactivity disorder-related intra-individual variability. Biol. Psychiatry 57, 1416-1423.

Chang, C., Cunningham, J. P., and Glover, G.H.(2009). Influence of heart rate on the BOLD signal: the cardiac response function. Neuroimage 44, 857-869.

Chang, C., and Glover, G. H. (2009). Effects of model-based physiological noise correction on default mode network anti-correlations and correlations. Neuroimage 47, 1448-1459.

Cherkassky, V. L., Kana, R. K., Keller, T. A., and Just, M. A. (2006). Functional connectivity in a baseline resting-state network in autism. Neuroreport 17, 1687-1690. 
Chugani, H. T., Phelps, M. E., and Mazziotta, J. C. (1987). Positron emission tomography study of human brain functional development. Ann. Neurol. 22, 487-497.

Church, J.A., Fair, D.A., Dosenbach, N.U., Cohen, A. L., Miezin, F. M., Petersen, S. E., and Schlaggar, B. L. (2009). Control networks in paediatric Tourette syndrome show immature and anomalous patterns of functional connectivity. Brain 132(Pt. 1), 225-238.

Cole, D. M., Smith, S. M., and Beckmann, C.F. (2010). Advances and pitfalls in the analysis and interpretation of restingstate FMRI data. Front. Syst. Neurosci. 4:8. doi:10.3389/fnsys.2010.00008.

Cordes, D., Haughton, V. M., Arfanakis, K., Carew, J. D., Turski, P.A., Moritz, C. H., Quigley, M. A., and Meyerand, M. E. (2001). Frequencies contributing to functional connectivity in the cerebral cortex in "resting-state" data. AJNR Am. J. Neuroradiol. 22, 1326-1333.

Cordes, D., Haughton, V. M., Arfanakis, K., Wendt, G. J., Turski, P. A., Moritz, C. H., Quigley, M. A., and Meyerand, M. E. (2000). Mapping functionally related regions of brain with functional connectivity MR imaging. AJNR Am. J. Neuroradiol. 21, 1636-1644.

Cullen, K. R., Gee, D. G., Klimes-Dougan, B., Gabbay, V., Hulvershorn, L., Mueller, B. A., Camchong, J., Bell, C. J., Houri, A., Kumra, S., Lim, K. O., Castellanos, F. X., and Milham, M. P. (2009). A preliminary study of functional connectivity in comorbid adolescent depression. Neurosci. Lett. 460, 227-231.

Damoiseaux, J. S., Rombouts, S. A., Barkhof, F., Scheltens, P., Stam, C. J., Smith, S. M., and Beckmann, C. F. (2006). Consistent resting-state networks across healthy subjects. Proc. Natl. Acad. Sci. U.S.A. 103, 13848-13853.

De Luca, M., Beckmann, C. F., De Stefano, N., Matthews, P. M., and Smith, S. M. (2006). fMRI resting state networks define distinct modes of long-distance interactions in the human brain. Neuroimage 29, 1359-1367.

Desjardins, A. E., Kiehl, K. A., and Liddle, P. F. (2001). Removal of confounding effects of global signal in functional MRI analyses. Neuroimage 13, 751-758.

Desmond, J. E., and Glover, G. H. (2002). Estimating sample size in functional MRI (fMRI) neuroimaging studies: statistical power analyses. J. Neurosci. Methods 118, 115-128.

Di Martino, A., Shehzad, Z., Kelly, C., Roy, A. K., Gee, D. G., Uddin, L. Q., Gotimer, K., Klein, D. F., Castellanos, F. X., and Milham, M. P. (2009). Relationship between cingulo-insular functional connectivity and autistic traits in neurotypical adults. Am. J. Psychiatry 166, 891-899.

Durston, S., Davidson, M. C., Tottenham, N., Galvan, A., Spicer, J., Fossella, J. A., and Casey, B. J. (2006). A shift from diffuse to focal cortical activity with development. Dev. Sci. 9, 1-8.

Fair, D.A., Cohen, A. L., Dosenbach, N.U., Church, J. A., Miezin, F. M., Barch, D. M., Raichle, M. E., Petersen, S. E., and Schlaggar, B. L. (2008). The maturing architecture of the brain's default network. Proc. Natl. Acad. Sci. U.S.A. 105, 4028-4032.

Fair, D. A., Cohen, A. L., Power, J. D. Dosenbach, N. U., Church, J. A., Miezin, F. M., Schlaggar, B. L., and Petersen, S. E. (2009). Functional brain networks develop from a "local to distributed” organization. PLoS Comput. Biol.5(5), e1000381.doi:10.1371/journal.pcbi.1000381.

Fair, D. A., Dosenbach, N. U., Church, J. A., Cohen, A. L., Brahmbhatt, S., Miezin, F. M., Barch, D. M., Raichle, M. E., Petersen, S. E., and Schlaggar, B. L. (2007a). Development of distinct control networks through segregation and integration. Proc. Natl. Acad. Sci. U.S.A. 104, 13507-13512.

Fair, D. A., Schlaggar, B. L., Cohen, A. L., Miezin, F. M., Dosenbach, N. U., Wenger, K. K., Fox, M. D., Snyder, A. Z., Raichle, M. E., and Petersen, S. E. (2007b). A method for using blocked and event-related fMRI data to study "resting state" functional connectivity. Neuroimage 35, 396-405.

Fox, M. D., and Raichle, M. E. (2007). Spontaneous fluctuations in brain activity observed with functional magnetic resonance imaging. Nat. Rev. Neurosci. 8, 700-711.

Fox, M. D., Zhang, D., Snyder, A. Z., and Raichle, M.E. (2009). The global signal and observed anticorrelated resting state brain networks. J. Neurophysiol. 101, 3270-3283.

Fransson, P., Skiold, B., Horsch, S., Nordell, A., Blennow, M., Lagercrantz, H., and Aden, U. (2007). Resting-state networks in the infant brain. Proc. Natl. Acad. Sci. U.S.A. 104, 15531-15536.

Frith, C. (2004). Is autism a disconnection disorder? Lancet Neurol. 3, 577.

Gao, W., Zhu, H., Giovanello, K. S., Smith, J. K., Shen, D., Gilmore, J.H., and Lin, W. (2009). Evidence on the emergence of the brain's default network from 2week-old to 2-year-old healthy pediatric subjects. Proc. Natl. Acad. Sci. U.S.A. 106, 6790-6795.

Giedd, J.N., Blumenthal, J., Molloy, E., and Castellanos, F.X. (2001). Brain imaging of attention deficit/hyperactivity disorder. Ann. N. Y. Acad. Sci. 931, 33-49.

Gogtay, N., Giedd, J. N., Lusk, L., Hayashi, K. M., Greenstein, D., Vaituzis, A. C., Nugent III, T. F., Herman, D. H.,
Clasen, L. S., Toga, A. W., Rapoport, J. L., and Thompson, P. M. (2004) Dynamic mapping of human cortical development during childhood through early adulthood. Proc. Natl. Acad. Sci. U. S. A. 101, 8174-8179.

Greicius, M. D., and Menon, V. (2004). Default-mode activity during a passive sensory task: uncoupled from deactivation but impacting activation. J. Cogn. Neurosci. 16, 1484-1492.

Hagmann, P., Cammoun, L., Gigandet, X., Meuli, R., Honey, C. J., Wedeen, V. J., and Sporns, O. (2008). Mapping the structural core of human cerebral cortex. PLoS Biol. 6, e159. doi:10.1371/ journal.pbio.0060159.

Harrison, B. J., Pujol, J., Lopez-Sola, M., Hernandez-Ribas, R., Deus, J., Ortiz, H., Soriano-Mas, C., Yucel, M., Pantelis, C., and Cardoner, N. (2008). Consistency and functional specialization in the default mode brain network. Proc. Natl. Acad. Sci. U.S.A. 105, 9781-9786.

He, B. J., Snyder, A. Z., Vincent, J. L., Epstein, A., Shulman, G. L., and Corbetta, M. (2007). Breakdown of functional connectivity in frontoparietal networks underlies behavioral deficits in spatial neglect. Neuron 53, 905-918.

Honey, C. J., Sporns, O., Cammoun, L., Gigandet, X., Thiran, J. P., Meuli, R., and Hagmann, P. (2009). Predicting human resting-state functional connectivity from structural connectivity. Proc. Natl. Acad. Sci. U.S.A. 106, 2035-2040.

Kelly, A. M., Di Martino, A., Uddin, L. Q., Shehzad, Z., Gee, D. G., Reiss, P. T., Margulies, D. S., Castellanos, F.X., and Milham, M. P. (2009). Development of anterior cingulate functional connectivity from late childhood to early adulthood. Cereb. Cortex 19, 640-657.

Kennedy, D. P., and Courchesne, E. (2008). The intrinsic functional organization of the brain is altered in autism. Neuroimage 39, 1877-1885.

Kiviniemi, V., Jauhiainen, J., Tervonen, O. Paakko, E., Oikarinen, J., Vainionpaa, V., Rantala, H., and Biswal, B. (2000). Slow vasomotor fluctuation in $\mathrm{fMRI}$ of anesthetized child brain. Magn. Reson. Med. 44, 373-378.

Kwon, H., Reiss, A. L., and Menon, V. (2002). Neural basis of protracted developmental changes in visuo-spatial working memory. Proc. Natl. Acad. Sci. U.S.A. 99, 13336-13341.

Lenroot, R. K., and Giedd, J. N. (2006) Brain development in children and adolescents: insights from anatomical magnetic resonance imaging. Neurosci. Biobehav. Rev. 30, 718-729.

Lewis, C. M., Baldassarre, A., Committeri, G., Romani, G. L., and Corbetta, M.
(2009). Learning sculpts the spontaneous activity of the resting human brain. Proc. Natl. Acad. Sci. U.S.A. 106, 17558-17563.

Lin, W., Zhu, Q., Gao, W., Chen, Y., Toh, C. H., Styner, M., Gerig, G., Smith, J. K. Biswal, B., and Gilmore, J. H. (2008). Functional connectivity MR imaging reveals cortical functional connectivity in the developing brain. AJNR Am. J. Neuroradiol. 29, 1883-1889.

Liu, W. C., Flax, J. F., Guise, K. G., Sukul, V., and Benasich,A.A. (2008). Functional connectivity of the sensorimotor area in naturally sleeping infants. Brain Res. 1223, 42-49.

Lowe, M. J., Mock, B. J., and Sorenson, J. A. (1998). Functional connectivity in single and multislice echoplanar imaging using resting-state fluctuations. Neuroimage 7, 119-132.

Luna, B., Thulborn, K. R., Munoz, D. P., Merriam, E. P., Garver, K. E., Minshew, N. J., Keshavan, M.S., Genovese, C. R., Eddy, W. F., and Sweeney, J. A. (2001). Maturation of widely distributed brain function subserves cognitive development. Neuroimage 13, 786-793.

Margulies, D. S., Kelly, A. M., Uddin, L. Q., Biswal, B. B., Castellanos, F. X., and Milham, M. P. (2007). Mapping the functional connectivity of anterior cingulate cortex. Neuroimage 37, 579-588.

Margulies, D. S., Vincent, J. L., Kelly, C., Lohmann, G., Uddin, L. Q., Biswal, B. B., Villringer, A., Castellanos, F. X., Milham, M. P., and Petrides, M. (2009). Precuneus shares intrinsic functional architecture in humans and monkeys. Proc. Natl. Acad. Sci. U.S.A. 106, 20069-20074.

Mazaika, P., Hoeft, F., Glover, G. H., and Reiss, A. L. (2009). Methods and software for fMRI analysis for clinical subjects. Paper presented at the Annual Meeting of the Organization for Human Brain Mapping.

Mazaika, P., Whitfield-Gabrieli, S., and Reiss, A. L. (2007). Artifact repair for fMRI data from high motion clinical subjects. Paper presented at the Annual Meeting of the Organization for Human Brain Mapping.

Meindl, T., Teipel, S., Elmouden, R., Mueller, S., Koch, W., Dietrich, O., Coates, U., Reiser, M., and Glaser, C. (2009). Testretest reproducibility of the defaultmode network in healthy individuals. Hum. Brain Mapp. 31, 237-46.

Monk, C. S., Peltier, S. J., Wiggins, J. L., Weng, S. J., Carrasco, M., Risi, S., and Lord, C. (2009). Abnormalities of intrinsic functional connectivity in autism spectrum disorders. Neuroimage 47, 764-772.

Murphy, K., Birn, R. M., Handwerker, D. A., Jones, T. B., and Bandettini, P. A. (2009). The impact of global signal 
regression on resting state correlations: are anti-correlated networks introduced? Neuroimage 44, 893-905.

Paakki, J.J., Rahko, J.,Long, X.Y., Moilanen, I., Tervonen, O., Nikkinen, J., Starck, T., Remes, J., Tuula, H., Haapsamo, H., Jussila, K., Kuusikko-Gauffin, S., Mattila, M.,Zang, Y., and Kiviniemi, V. (2010). Alterations in regional homogeneity of resting-state brain activity in autism spectrum disorders. Brain Res. 1321, 169-179.

Raichle, M. E. (2010). Two views of brain function. Trends Cogn. Sci. 14, 180-190.

Raichle, M. E., MacLeod, A. M., Snyder, A. Z., Powers, W. J., Gusnard, D. A., and Shulman, G.L. (2001). A default mode of brain function. Proc. Natl. Acad. Sci. U.S.A. 98, 676-682.

Redcay, E., Kennedy,D.P., and Courchesne, E. (2007). fMRI during natural sleep as a method to study brain function during early childhood. Neuroimage 38, 696-707.

Rivera, S. M., Reiss, A. L., Eckert, M. A., and Menon, V.(2005). Developmental changes in mental arithmetic: evidence for increased functional specialization in the left inferior parietal cortex. Cereb. Cortex 15, 1779-1790.

Shehzad, Z., Kelly, A. M., Reiss, P. T., Gee, D. G., Gotimer, K., Uddin, L. Q., Lee, S., Margulies, D. S., Roy, A. K., Biswal, B. B., Petkova, E., Castellanos, F. X., and Milham, M. P. (2009). The resting brain: unconstrained yet reliable. Cereb. Cortex 19, 2209-2229.

Shulman, G. L., Fiez, J. A., Corbetta, M., Buckner, R. L., Miezen, F. M., Raichle, M. E., and Petersen, S. E. (1997). Common blood flow changes across visual tasks: II. Decreases in cerebral cortex. J. Cogn. Neurosci. 9, 648-663.

Smith, S. M., Fox, P. T., Miller, K. L., Glahn, D. C., Fox, P. M., Mackay, C. E., Filippini, N., Watkins, K. E., Toro, R., Laird, A. R., and Beckmann, C. F. (2009). Correspondence of the brain's functional architecture during activation and rest. Proc. Natl. Acad. Sci. U.S.A. 106, 13040-13045.

Sonuga-Barke, E. J., and Castellanos, F. X. (2007). Spontaneous attentional fluctuations in impaired states and pathological conditions: a neurobiological hypothesis. Neurosci. Biobehav. Rev. 31, 977-986.

Sowell, E. R., Thompson, P. M., Leonard, C. M., Welcome, S. E., Kan, E., and
Toga, A. W. (2004). Longitudinal mapping of cortical thickness and brain growth in normal children. $J$. Neurosci. 24, 8223-8231.

Spreng, R. N., Mar, R. A., and Kim, A. S. (2009). The common neural basis of autobiographical memory, prospection, navigation, theory of mind, and the default mode: a quantitative meta-analysis. J. Cogn. Neurosci. 21, 489-510.

Stevens, M.C. (2009). The developmental cognitive neuroscience of functional connectivity. Brain Cogn. 70, 1-12.

Stevens, M. C., Pearlson, G. D., and Calhoun, V.D. (2009). Changes in the interaction of resting-state neural networks from adolescence to adulthood. Hum. Brain Mapp. 30, 2356-2366.

Supekar, K., Musen, M., and Menon, V. (2009). Development of large-scale functional brain networks in children. PLoS Biol. 7, e1000157. doi:10.1371/ journal.pbio.1000157.

Supekar, K., Uddin, L. Q., Prater, K., Amin, H., Greicius, M. D., and Menon, V. (2010). Development of functional and structural connectivity within the default mode network in young children. Neuroimage (in press).

Tamm, L., Menon, V., and Reiss, A. L. (2002). Maturation of brain function associated with response inhibition. J. Am. Acad. Child Adolesc. Psychiatry 41, 1231-1238.

Thomas, K. M., Hunt, R. H., Vizueta, N., Sommer, T., Durston, S., Yang, Y., and Worden, M. S. (2004). Evidence of developmental differences in implicit sequence learning: an fMRI study of children and adults. J. Cogn. Neurosci. 16, 1339-1351.

Thomason, M. E., Chang, C. E., Glover, G. H., Gabrieli, J. D., Grecius, M. D., and Gotlib, I. H. (2008). Defaultmode function and task-induced deactivation have overlapping brain substrates in children. Neuroimage 41, 1493-1503.

Thomason, M. E., Yoo, D. J., Glover, G. H., and Gotlib, I. H. (2009). BDNF genotype modulates resting functional connectivity in children. Front. Hum. Neurosci. 3:55. doi: 10.3389/neuro.09.055.2009.

Tian, L., Jiang, T., Liang, M., Zang, Y., He, Y., Sui, M., and Wang, Y. (2008). Enhanced resting-state brain activities in ADHD patients: a fMRI study. Brain Dev. 30, 342-348.
Tian, L., Jiang, T., Wang, Y., Zang, Y., He, Y., Liang, M., Sui, M., Cao, Q., Hu, S., Peng, M., and Zhou, Y. (2006). Altered restingstate functional connectivity patterns of anterior cingulate cortex in adolescents with attention deficit hyperactivity disorder. Neurosci. Lett. 400, 39-43.

Uddin, L. Q., Iacoboni, M., Lange, C., and Keenan, J. P. (2007). The self and social cognition: the role of cortical midline structures and mirror neurons. Trends Cogn. Sci. 11, 153-157.

Uddin, L. Q., Kelly, A. M. C., Biswal, B. B., Margulies, D. S., Shehzad, Z., Shaw, D., Ghaffari, M., Rotrosen, J., Adler, L. A., Castellanos, F. X., and Milham, M. P. (2008). Network homogeneity reveals decreased integrity of defaultmode network in ADHD. J. Neurosci. Methods 169, 249-254.

Uddin, L. Q., and Menon, V. (2009). The anterior insula in autism: under-connected and under-examined. Neurosci. Biobehav. Rev. 33, 1198-1203.

van den Heuvel, M. P., Mandl, R. C., Kahn, R. S., and Hulshoff Pol, H. E. (2009). Functionally linked restingstate networks reflect the underlying structural connectivity architecture of the human brain. Hum. Brain Mapp. 30, 3127-3141.

van de Ven, V.G., Formisano, E., Prvulovic, D., Roeder, C. H., and Linden, D. E. (2004). Functional connectivity as revealed by spatial independent component analysis of fMRI measurements during rest. Hum. Brain Mapp. 22, 165-178.

Van Dijk, K. R.,Hedden, T., Venkataraman, A., Evans, K. C., Lazar, S. W., and Buckner, R. L. (2009). Intrinsic functional connectivity as a tool for human connectomics: theory, properties, and optimization. J. Neurophysiol. 103, 297-321.

Weissenbacher, A., Kasess, C., Gerstl, F., Lanzenberger, R., Moser, E. and Windischberber, C. (2009). Correlations and anticorrelations in resting-state functional connectivity MRI: a quantitative comparison of preprocessing strategies. Neuroimage 47, 1408-1416.

Weng, S. J., Wiggins, J. L., Peltier, S. J., Carrasco, M., Risi, S., Lord, C., and Monk, C. S. (2010). Alterations of resting state functional connectivity in the default network in adolescents with autism spectrum disorders. Brain Res. 1313, 202-214.
Yerys, B. E., Jankowski, K. F., Shook, D., Rosenberger, L. R., Barnes, K. A., Berl, M. M., Ritzl, E. K., VanMeter, J., Vaidya, C. J., and Gaillard, W. D. (2009). The fMRI success rate of children and adolescents: typical development, epilepsy, attention deficit/hyperactivity disorder, and autism spectrum disorders. Hum. Brain Mapp. 30, 3426-3435.

Zang, Y. F., Yong, H., Chao-Zhe, Z., Qing-Jiu, C., Man-Qiu, S., Meng, L., Li-Xia, T., Tian-Zi, J., and Yu-Feng, W. (2007). Altered baseline brain activity in children with ADHD revealed by resting-state functional MRI. Brain Dev. 29, 83-91.

Zhu, C.Z., Zang, Y.F., Cao, Q. J., Yan, C. G., He, Y., Jiang, T. Z., Sui, M., and Wang, Y. (2008). Fisher discriminative analysis of resting-state brain function for attention-deficit/hyperactivity disorder. Neuroimage 40, 110-120.

Zuo, X. N., Di Martino, A., Kelly, C., Shehzad, Z. E., Gee, D. G., Klein, D. F., Castellanos, F. X., Biswal, B. B., and Milham, M. P. (2010a). The oscillating brain: complex and reliable. Neuroimage 49, 1432-1445.

Zuo, X. N., Kelly, C., Adelstein, J.S., Klein, D. F., Castellanos, F. X., and Milham, M.P. (2010b). Reliable intrinsic connectivity networks: test-retest evaluation using ICA and dual regression approach. Neuroimage 49, 2163-2177.

Conflict of Interest Statement: The authors declare that the research was conducted in the absence of any commercial or financial relationships that could be construed as a potential conflict of interest.

Received: 10 February 2010; paper pending published: 10 March 2010; accepted: 16 April 2010; published online: 21 May 2010.

Citation: Uddin LQ, Supekar K and Menon V (2010) Typical and atypical development of functional human brain networks: insights from resting-state fMRI. Front. Syst. Neurosci. 4:21. doi: 10.3389/fnsys.2010.00021

Copyright (c) 2010 Uddin, Supekar and Menon. This is an open-access article subject to an exclusive license agreement between the authors and the Frontiers Research Foundation, which permits unrestricted use, distribution, and reproduction in any medium, provided the original authors and source are credited. 